\title{
The PHSRN sequence induces extracellular matrix invasion and accelerates wound healing in obese diabetic mice
}

\author{
Donna L. Livant, ${ }^{1}$ R. Kaye Brabec, ${ }^{1}$ Kotoku Kurachi, ${ }^{2}$ David L. Allen, ${ }^{2}$ Yanling Wu, ${ }^{2}$ \\ Ronald Haaseth, ${ }^{3}$ Philip Andrews, ${ }^{3,4}$ Stephen P. Ethier, ${ }^{5}$ and Sonja Markwart ${ }^{1}$ \\ ${ }^{1}$ Department of Cell and Developmental Biology, \\ ${ }^{2}$ Department of Human Genetics, \\ ${ }^{3}$ Protein and Carbohydrate Structure Facility, \\ ${ }^{4}$ Department of Biochemistry, and \\ ${ }^{5}$ Department of Radiation Oncology, University of Michigan Medical School, Ann Arbor, Michigan, USA \\ Address correspondence to: Donna L. Livant, Department of Cell and Developmental Biology, \\ University of Michigan Medical School, 1150 West Medical Center Drive, Ann Arbor, Michigan 48109-0616, USA. \\ Phone: (734) 764-0313; Fax: (734) 763-1166; E-mail: dlivant@umich.edu. \\ Received for publication September 22, 1999, and accepted in revised form April 7, 2000.
}

The PHSRN sequence of the plasma fibronectin ( $\mathrm{pFn}$ ) cell-binding domain induces human keratinocytes and fibroblasts to invade the naturally serum-free extracellular matricies of sea urchin embryos. The potency of acetylated, amidated PHSRN (Ac-PHSRN-NH ${ }_{2}$ ) is significantly increased, making it more active on a molar basis than the $120-\mathrm{kDa}$ cell-binding domain of $\mathrm{pFn}$. Arginine is important to this activity because PHSAN and PHSEN are inactive, as is a randomized sequence peptide, Ac-HSPNR-NH $\mathrm{N}_{2}$. One treatment with Ac-PHSRN-NH $\mathrm{N}_{2}$ stimulates reepithelialization and contraction of dermal wounds in healing-impaired, obese diabetic C57BL6/KsJ $d b / d b$ mice. Wound closure is equally rapid in treated $d b / d b$ and $d b /+$ mice and may be more rapid than in untreated nondiabetic $d b /+$ littermates. In contrast, treatment with either Ac-HSPNR- $\mathrm{NH}_{2}$ or normal saline (NS) has no effect. Analysis of sectioned $d b / d b$ wounds shows that, in contrast to treatment with Ac-HSPNR$\mathrm{NH}_{2}$ or NS, a single Ac-PHSRN-NH $\mathrm{N}_{2}$ treatment stimulates keratinocyte and fibroblast migration into wounds, enhances fibroplasia and vascularization in the provisional matrix, and stimulates the formation of prominent fibers that may be associated with wound contraction.

J. Clin. Invest. 105:1537-1545 (2000).

\section{Introduction}

Rapid induction of keratinocyte and fibroblast migration into wounds is necessary for tissue repair. Reepithelialization begins soon after injury as epidermal cells become migratory $(1,2)$. Because plasma fibronectin $(\mathrm{pFn})$ circulates in the blood at $0.3-0.5 \mathrm{mg} / \mathrm{mL}$ (reviewed in ref. 3) and binds to fibrin, it is a significant component of clot provisional matrix. If the basement membrane is destroyed, epidermal cells migrate over the $\mathrm{pFn}$ rich provisional matrix (4-6). The fibronectin of the provisional matrix is also known to promote the movement of fibroblasts, macrophages, and blood vessels into the wound space $(1,7)$. Keratinocytes, other epithelial cell types such as mammary and prostate epithelial cells, and fibroblasts express the $\alpha 5 \beta 1$ integrin fibronectin receptor (8-11). Although keratinocytes express less $\alpha 5 \beta 1$ than $\alpha 3 \beta 1, \alpha 5 \beta 1$ is more important for migration (8). Also, expression of $\alpha 5 \beta 1(12,13)$ and specific metalloproteinases (14) are increased in motile keratinocytes at the leading edge of the new epithelium. Keratinocyte and fibroblast cell movement is supported by $\mathrm{pFn}(15$, 16). Fragments of $\mathrm{pFn}$ containing the cell-binding domain, which are found in wounds due to localized proteinase activity $(17,18)$, stimulate monocyte $(7)$ and fibroblast chemotaxis through the dermis (19-21), as well as matrix metalloproteinase expression by fibrob- lasts (11). This interaction also stimulates cellular invasion of the provisional matrix and wound stroma (16; reviewed in ref. 22). The pFn cell-binding domain also induces monocytes to extravasate from proximal vessels, enabling them to enter the wound and differentiate into inflammatory macrophages (23), and can stimulate normal human endothelial cell motility in vitro (24). Thus, the invasive/migratory phenotype expressed by epithelial cells and fibroblasts after wounding may result from $p F n$ fragmentation and $\alpha 5 \beta 1$ integrin-mediated signaling. Furthermore, this interaction promotes the immigration or motility of a number of cell types involved in healing through a variety of extracellular matrices, including the stroma as well as vascular basement membranes $(23,24)$.

To define the invasion-stimulatory sequences of $\mathrm{pFn}$, the naturally serum-free basement membranes and associated extracellular matrices of sea urchin embryos (SUECM) were used as in vitro invasion substrates (25). We report that the PHSRN sequence of pFn stimulates SUECM invasion by keratinocytes and fibroblasts. Acetylated and amidated PHSRN peptide (Ac-PHSRN-NH is greatly increased in activity, whereas a randomized sequence peptide, Ac-HSPNR-NH ${ }_{2}$, is inactive. In derivatives of the PHSRN peptide, substitution of arginine by alanine or glutamic acid also results in inactivity. 
Topical application of Ac-PHSRN- $\mathrm{NH}_{2}$ accelerates the healing of dermal wounds in genetically obese, diabetic C57BL6/KsJ $d b / d b$ mice, resulting in wound closure that may be faster than that of untreated wounds in nondiabetic C57BL6/KsJ db/+ littermates, and as fast as that of Ac-PHSRN-NH$H_{2}$-treated wounds in $d b /+$ mice. In contrast, neither Ac-HSPNR-NH $\mathrm{N}_{2}$ nor normal saline (NS) treatment stimulates $d b / d b$ wound healing. Comparison of sectioned Ac-PHSRN-NH$H_{2}$, Ac-HSPNR$\mathrm{NH}_{2}$, and NS-treated $d b / d b$ wounds for 8 days after wounding shows that Ac-PHSRN-NH $\mathrm{N}_{2}$ treatment stimulates keratinocyte migration across the developing granulation tissue, as well as fibroblast immigration into the provisional matrix.

\section{Methods}

Cell culture and SU-ECM invasion assays. In SU-ECM invasion substrates, a basement membrane surrounds a blastocele in which invading cells localize shortly after suspension and placement on the outer surfaces. Even in the presence of serum, these invasion substrates have been shown to be free of the background invasion by normal cells (25) that is observed when artificial or reconstituted basement membranes are used $(26,27)$. Because they are naturally serum free, SU-ECM have recently been used to define a potent competitive inhibitor of $\mathrm{pFn}$-induced invasion by metastatic prostate cancer cells; this inhibitor appears to be an effective anti-tumorigenic and anti-metastatic agent in Copenhagen rats (28).

In vitro invasion assays on SU-ECM were performed with or without added FCS, as described previously (25). Undifferentiated, first or second passage human keratinocytes (a gift of C. Marcelo, University of Michigan, Ann Arbor, Michigan, USA) were cultured in serum-free keratinocyte growth medium (Clonetics Corp., San Diego, California, USA). First or second passage neonatal foreskin fibroblasts (a gift of J. Varani, University of Michigan, Ann Arbor, Michigan, USA) were cultured as described (25). Cells from single-cell suspensions made with $0.25 \%$ trypsin/EDTA (GIBCO BRL, Grand Island, New York, USA) were placed on SU-ECM invasion substrates with or without FCS for 4 hours at $37^{\circ} \mathrm{C}$, the time required to observe maximal invasion percentages. Peptides were present at a uniform concentration in these assays, rather than in a gradient. Chemotaxis results from gradients in inductive signaling, often created by gradients in ligand concentration. However, as assessed by fluorescent antibody staining, short-term asymmetric distribution of the fibronectin receptors results from trypsinization to suspend the cells just before assay (data not shown). As indicated by the maximal invasion percentage of about $25 \%$, which is always reached in $3-4$ hours $(25,28$, and data not shown), this asymmetric distribution probably results in differential signaling across the cell surface to stimulate invasion by appropriately oriented cells. Cellular viability in SU-ECM invasion assays ranged from $90 \%$ to $99 \%$ before fixation in $2 \%$ formaldehyde and scoring of invasion percentages. Inva- sion percentages are the ratio of the number of cells located in the blastocelic cavities of SU-ECM invasion substrates to the total number of single cells adhering to their exterior and interior surfaces. Each invasion percentage was the result of three or four independent determinations involving the scoring of the positions of all individual cells adhering to SU-ECM, typically about 100 individual cells for each determination. Before use, the intactness of the pFn $120-\mathrm{kDa}$ chymotryptic fragment (Sigma Biochemicals and Regents for Life Science Research, St. Louis, Missouri, USA) was verified by denaturing gel electrophoresis (29).

Inbibition of epithelial cell and fibroblast invasion with antiintegrin antibodies. P1D6 or P1B5 monoclonal antibodies $(30,31)$, purchased from Oncogene Research Products (Cambridge, Massachusetts, USA), were bound to keratinocytes or to human foreskin fibroblasts (HFFs) in serum-containing medium on ice for 30 minutes before or after a 5-minute exposure of the cells to 60 $\mu \mathrm{g} / \mathrm{mL}$ of the $120-\mathrm{kDa} \mathrm{pFn}$ cell-binding domain at room temperature. Prebound cells were diluted 20 -fold, then dropped as cell suspensions on SU-ECM in the appropriate medium with FCS.

Peptide synthesis. The following peptides were synthesized using standard Fmoc/t-butyl protection strategies (32) at $25 \mu \mathrm{M}$ and $100 \mu \mathrm{M}$ scales on a Rainin Symphony (Rainin Instrument Co., Woburn, Massachusetts, USA) multiple peptide synthesizer: PHSRN, amino-terminal acetylated and carboxy-terminal amidated PHSRN (AcPHSRN-NH$H_{2}$ ), the randomized sequence control (AcHSPNR-NH $\mathrm{N}_{2}$, PHSAN, PHSEN, and PHSKN. Larger scales ( $1 \mathrm{mmol}$ and above) were synthesized on a Rainin Sonata large-scale synthesizer. Peptides were synthesized on chlorotrityl resin or on Rink resin. The completed peptides were cleaved from the resin support and the side chain protecting groups were simultaneously removed by trifluoroacetic acid (TFA). The peptides were then precipitated with diethyl ether, purified by preparative HPLC, and then lyophilized. Peptide purities were assessed by reverse phase HPLC, performed according to standard procedures (33), as 92\% for PHSRN, 95\% for Ac-PHSRN-NH ${ }_{2}$, 97\% for PHSAN, 95\% for PHSEN, 93\% for PHSKN, and 95\% for Ac-HSPNR-NH $\mathrm{N}_{2}$ (not shown). Peptide structures were confirmed by mass spectrometry and amino acid analysis (not shown). Because the presence of TFA, a very strong acid, could affect peptide activity in aqueous solution or injure the mice, residual TFA was removed by gel permeation chromatography on a Sephadex G-10 column equilibrated with $1 \mathrm{~N}$ acetic acid. Peptides were then lyophilized to remove the excess acetic acid and were stored as lyophilized solids at $-20^{\circ} \mathrm{C}$ until solubilization in NS at $1 \mathrm{mg} / \mathrm{mL}$ for use in vitro or in mouse wounds.

Motility assays. Standard checkerboard analyses were performed to compare the effects of the PHSRN peptide on chemotaxis and chemokinesis in Boyden chambers with $8-\mu \mathrm{m}$ pores for HFFs (Becton Dickinson Labware, Bedford, Massachusetts, USA), and with $12-\mu \mathrm{m}$ pores (Sigma) for keratinocytes. Chambers were coated with 
gelatin by allowing sterile $0.2 \%$ gelatin to sit in the chambers for $3-5$ minutes before drying at $37^{\circ} \mathrm{C}$ for $1-2$ hours. Cells were preincubated in serum-free medium with either $2 \mu \mathrm{M}$ PHSRN or $0.2 \%$ BSA for 1 hour. Cells were then suspended by trypsinization and placed in the upper chambers in serum-free medium containing either $0.2 \%$ BSA or $2 \mu \mathrm{M}$ PHSRN peptide. Two-micromolar PHSRN or $0.2 \%$ BSA was placed in the lower chambers. Serum-free basal medium (Clonetics Corp.) was used for keratinocytes; serum-free DMEM (GIBCO BRL) was used for HFFs. All assays also used a Boyden chamber with $10 \%$ FCS in the lower well as a positive control. Motility assays were performed according to standard procedures (34). All cells were allowed to migrate for 12 hours before microscopic scoring of at least ten randomly chosen fields per chamber.

Wounding of $d b / d b$ mice and their normal littermates for measurements of wound areas. Biopsy punches $4 \mathrm{~mm}$ in diameter (Henry Schein Inc., Port Washington, New York, USA) were used to wound C57BL6/KsJ $d b / d b$ and C57BL6/KsJ $d b /+$ mice (35-38), aged 3-7 months (The Jackson Laboratory, Bar Harbor, Maine, USA). Anesthetized mice were wounded once or twice on the upper back by pinching the skin away from the underlying fascia and muscle and pushing the biopsy punch through the skin as it lay over a forefinger. As judged by measuring sections of day-old wounds with a reticle at 400 -fold magnification, $d b / d b$ mice were wounded to a mean depth of $1.7 \mathrm{~mm}( \pm 0.4 \mathrm{~mm}, \mathrm{SD})$, with a range of $1.3-2.2$ $\mathrm{mm}$. The depth of the wounds in the $d b /+$ mice appeared to be somewhat less $(1.1-1.5 \mathrm{~mm})$ because of the reduced amount of subcutaneous fat in these animals. However, in no case was the underlying muscle wounded. In each experiment, the mice were age matched. To evaluate wound area vs. time in AcPHSRN-NH ${ }_{2}$-treated, Ac-HSPNR-NH ${ }_{2}$-treated, or NStreated $d b / d b$ mice, as well as in Ac-PHSRN-NH $\mathrm{N}_{2}$-treated or NS-treated $d b /+$ mice, C57BL6/KsJ $d b / d b$ mice $(n=100)$ and $d b /+$ nondiabetic littermates $(n=80)$, aged 3-7 months were wounded in duplicate as described above. In some $d b / d b$ mice $(n=60)$, both wounds in each mouse received a single identical treatment: $2 \mu \mathrm{g}$ AcPHSRN-NH $H_{2}$ or $2 \mu \mathrm{g}$ Ac-HSPNR-NH $\mathrm{N}_{2}$ in $5 \mu \mathrm{L}$ NS were used in three groups of ten mice each. NS only $(5 \mu \mathrm{L})$ was used to treat the duplicate wounds of other $d b / d b$ mice $(n=20)$. To assess the locality of the effect of AcPHSRN-NH ${ }_{2}$ treatment, the remaining $d b / d b$ mice $(n=$ 20) received $2 \mu \mathrm{g}$ Ac-PHSRN-NH$H_{2}$ or Ac-HSPNR- $\mathrm{NH}_{2}$ in one of two duplicate wounds; the remaining wound on each mouse received $5 \mu \mathrm{L}$ NS only. Eighty $d b /+$ mice were wounded in duplicate, as described for the $d b / d b$ mice. In some $d b /+$ mice $(n=30)$, both wounds received $2 \mu \mathrm{g}$ Ac-PHSRN-NH ${ }_{2}$ in $5 \mu$ L NS. In other $d b /+$ mice $(n$ $=30$ ), both wounds received only NS. To assess the locality of the effect of Ac-PHSRN-NH$H_{2}$ treatment, the remaining $d b /+$ mice $(n=20)$ received $2 \mu$ g Ac-PHSRN$\mathrm{NH}_{2}$ or Ac-HSPNR-NH $\mathrm{NH}_{2}$ in one of two duplicate wounds; the remaining wound on each mouse received $5 \mu \mathrm{L}$ NS only. Mean wound areas were determined as described (38). Peptide solutions and controls were coded before use by individuals not involved in the wounding or in the scoring of wound areas.

Histological analysis of Ac-PHSRN-NH${ }_{2}, A c-H S P N R-\mathrm{NH}_{2}$, and NS-treated $d b / d b$ wounds. On day $0,24 d b / d b$ mice aged 3 months were wounded in duplicate with a 4-mm biopsy punch as described above, and were divided into three treatment groups of 8 mice each. The wounds of each group were treated once with $5 \mu \mathrm{L}$ NS containing either $2 \mu \mathrm{g}$ Ac-PHSRN-NH$H_{2}$ or $2 \mu \mathrm{g}$ Ac-HSPNR-NH $\mathrm{N}_{2}$, or once with NS alone. On days 1 through 8 , a mouse from each treatment group was sacrificed. Its wounds were dissected out, each surrounded by unwounded skin. Thus, all the wounded mice were assayed in this experiment. Wounds were fixed for at least 3 days in 5\% formaldehyde in PBS, and then embedded in paraffin. Before sectioning and staining with hematoxylin and eosin according to standard procedures (39), the blocks were cut to expose the wounded tissue near the center of each wound. For each wound, the distance keratinocytes migrated from the wound edges was measured in five groups of three serial $5-\mu \mathrm{m}$ sections. Fifty sections, or $250 \mu \mathrm{m}$, separated each group of three serial sections from the next group. The distance migrated was measured using a reticle and a standard grid with phase-contrast optics at 100-fold and 250-fold magnification. The entire wound bed or new dermal tissue was also examined in each section of each wound at 200-fold and 400-fold magnification. Sections were photographed at 400-fold magnification.

\section{Results}

Invasion induction by the PHSRN sequence of the pFn cellbinding domain. The effect of the $120-\mathrm{kDa}$ pFn fragment on keratinocyte and fibroblast invasion was assessed using SU-ECM invasion substrates in the presence and absence of FCS. Figure 1 shows the mean percentages of invaded cells in response to various fragment concentrations. Irrespective of the presence of FCS in the invasion assay media, keratinocytes and fibroblasts were

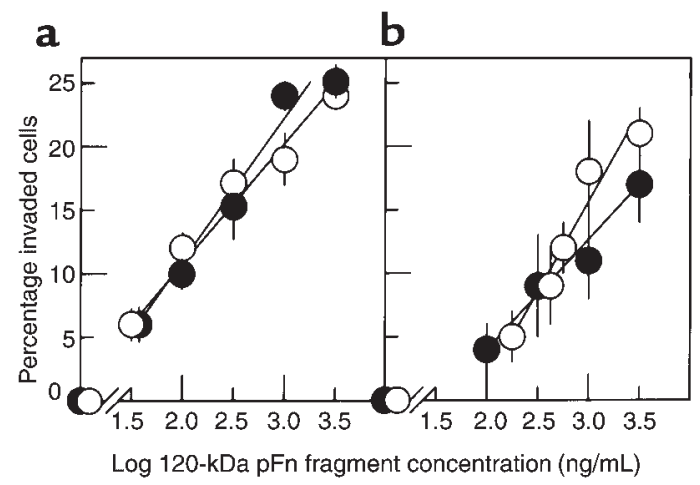

Figure 1

Invasive responses of normal keratinocytes and fibroblasts to the 120-kDa pFn fragment. (a) Undifferentiated keratinocytes. (b) Fibroblasts. White circles, serum-free media; black circles, FCS-containing media. Data presented as mean \pm SD. 


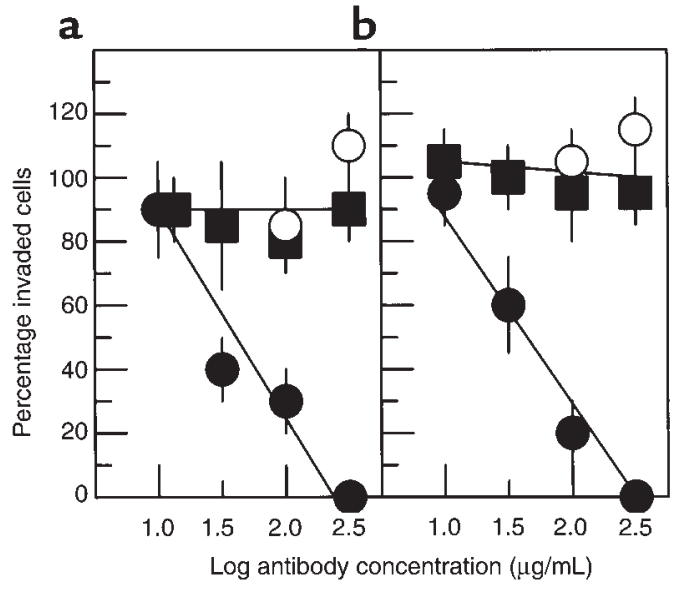

Figure 2

Effects of P1D6 anti- $\alpha 5 \beta 1$ or P1B5 anti- $\alpha 3 \beta 1$ antibodies on invasion. (a) Keratinocytes. (b) Fibroblasts. Y-axis shows mean percentage of invaded cells $( \pm S D)$ relative to the mean percentage invaded without antibody. Black circles, P1D6 prebound to cells before exposure to $10 \mu \mathrm{g} / \mathrm{mL} 120-\mathrm{kDa} \mathrm{pFn}$ fragment and placement on SU-ECM invasion substrates. White circles, P1D6 bound to cells in the presence of $120-\mathrm{kDa}$ fragment. Black squares, P1B5 bound to cells before exposure to the $120-\mathrm{kDa}$ pFn fragment.

stimulated to invade by the $120-\mathrm{kDa}$ pFn fragment, with similar dose-response relationships. No invasion occurred in the absence of the fragment in the presence or absence of serum.

Because the $\alpha 5 \beta 1$ fibronectin receptor is known to interact with the PHSRN and RGD sequences of the cell-binding domain $(40,41)$, invasion induction by the $120-\mathrm{kDa} \mathrm{pFn}$ fragment suggested the possible involvement of $\alpha 5 \beta 1$ integrin. Keratinocytes and fibroblasts were suspended and bound to the monoclonal anti$\alpha 5 \beta 1$ P1D6 function-blocking antibody, either before invasion stimulation by the $120-\mathrm{kDa} \mathrm{pFn}$ fragment or concurrently. The epitope of the P1D6 antibody has been shown to include the specific pocket of the $\alpha 5$ chain that binds the PHSRN sequence of the cell-binding domain (42). Although the presence of the $120-\mathrm{kDa}$ $\mathrm{pFn}$ fragment varied during antibody binding, incubation on SU-ECM to detect invasion always occurred in the presence of the fragment. Keratinocytes (8) and fibroblasts (43) express $\alpha 3 \beta 1$, which also interacts with $\mathrm{pFn}$. Therefore, as specificity controls, the cells were prebound to the function-blocking, anti- $\alpha 3 \beta 1 \mathrm{P} 1 \mathrm{~B} 5$ monoclonal antibody before invasion induction. As shown in Figure 2, P1D6 efficiently blocked invasion by both cell types when bound to cells before invasion induction by the $120-\mathrm{kDa} \mathrm{pFn}$ fragment, whereas the fraction of cells adhered to SU-ECM appeared to be unaffected. However, a high concentration of P1D6 failed to inhibit invasion induction by the $120-\mathrm{kDa}$ fragment when antibody binding occurred after fragment exposure of the cells. In contrast, binding high concentrations of P1B5 to cells before exposure to the $120-\mathrm{kDa}$ pFn fragment did not inhibit fragmentinduced invasion. Thus, $\alpha 5 \beta 1$ integrin was identified as the receptor for invasion induction by the $120-\mathrm{kDa}$ cell-binding domain, which probably occurred before SU-ECM adhesion. These results are consistent with the observation that the PHSRN-containing synergy region of the $120-\mathrm{kDa}$ pFn cell-binding domain blocks P1D6 antibody binding to $\alpha 5 \beta 1$ (42). The results suggest that the P1D6 epitope coincides with the region of $\alpha 5 \beta 1$ interacting with the invasion-inducing sequences of $\mathrm{PFn}$, and implicate the PHSRN sequence as an invasion inducer for epithelial cells and fibroblasts.

Invasion induction by the PHSRN peptide. The induction of keratinocyte and fibroblast invasion by derivatives of the PHSRN sequence was evaluated on SU-ECM at peptide concentrations of $10-1,000 \mathrm{ng} / \mathrm{mL}$ ( $20 \mathrm{nM}$ to $2 \mu \mathrm{M})$. As shown in Figure 3, these cells exhibited similar log-linear responses to the PHSRN peptide. The invasion-inducing activity of Ac-PHSRN- $\mathrm{NH}_{2}$ was increased by about 100 -fold for both keratinocytes and fibroblasts. The lack of invasion-inducing activity of Ac-HSPNR-NH $\mathrm{H}_{2}$ at concentrations as high as $10 \mu \mathrm{g} / \mathrm{mL}$ $(20 \mu \mathrm{M})$ indicated the sequence specificity of this effect. Consistent with its ability to stimulate a motile response, the presence of the PHSRN peptide in solution has been shown to stimulate dissociation of adherent cells from the substratum; and substitution of amino acids with neutral or acidic side chains for arginine has been shown to abrogate this activity (40). Because invasion involves a change in substrate adhesion, the importance of the positively charged arginine side chain for keratinocyte and fibroblast invasion induction was evaluated. As shown in Figure 3, the PHSAN and PHSEN peptides, which have a neutral side chain of alanine or a negatively charged side chain of glutamic acid at the position of the arginine side chain, were completely inactive at concentrations as high as $10 \mu \mathrm{g} / \mathrm{mL}$. However, the PHSKN peptide, in which lysine is substituted for arginine, had greatly

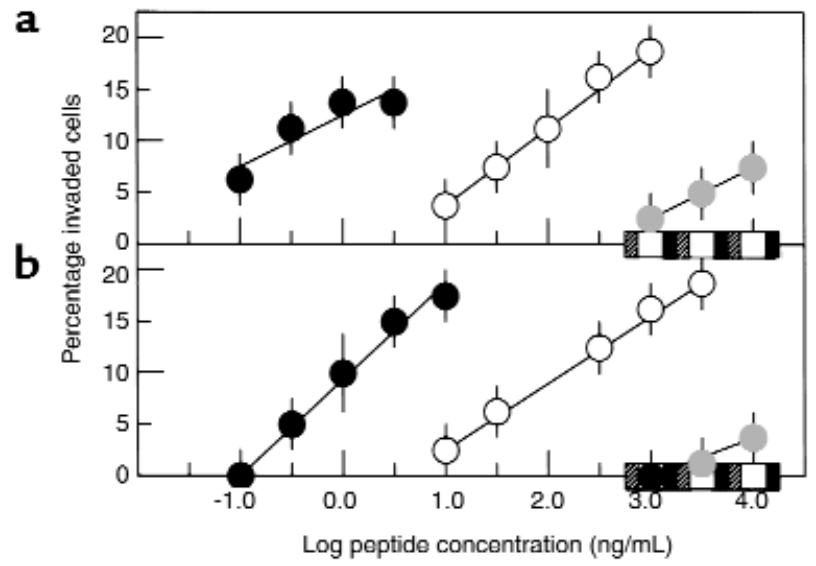

Figure 3

Invasive responses of keratinocytes and fibroblasts to derivatives of the PHSRN sequence. (a) Keratinocytes. (b) Fibroblasts. Black circles, Ac-PHSRN-NH ${ }_{2}$; white circles, PHSRN; gray circles, PHSKN; black squares, PHSAN; white squares, PHSEN; hatched squares, Ac$\mathrm{HSPNR}-\mathrm{NH}_{2}$. Data presented as mean $\pm \mathrm{SD}$. 


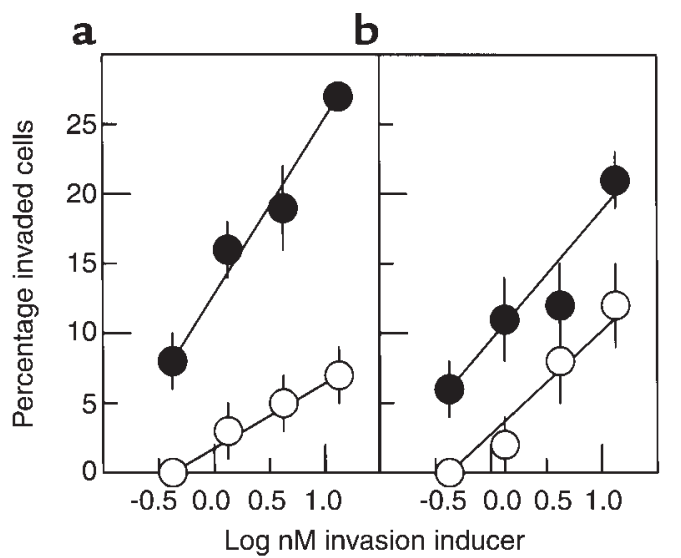

Figure 4

Comparative dose-response curves of Ac-PHSRN-NH $\mathrm{H}_{2}$ and the 120kDa pFn fragment. (a) Keratinocytes. (b) Fibroblasts. Y-axis shows mean percentages of invaded cells $( \pm S D)$. Black circles, Ac-PHSRN$\mathrm{NH}_{2}$; white circles, 120-kDa pFn fragment.

reduced but detectable invasion-inducing activity. This suggested that a positively-charged side chain at the fourth position may be necessary but not sufficient for invasion induction, and that other structural features of the arginine side chain could also be important.

Because Ac-PHSRN-NH $\mathrm{H}_{2}$ had 100-fold greater invasion-inducing activity than PHSRN, it was thought possible that Ac-PHSRN-NH$H_{2}$ might be more potent at inducing invasion than the intact $120-\mathrm{kDa}$ pFn cellbinding domain. To test this, keratinocytes and fibroblasts were induced to invade SU-ECM by identical molar concentrations of Ac-PHSRN-NH $\mathrm{NH}_{2}$ or the $120-\mathrm{kDa} \mathrm{pFn}$ fragment in parallel invasion assays using the same sets of SU-ECM invasion substrates. As shown in Figure 4, Ac-PHSRN-NH $\mathrm{N}_{2}$ was consistently at least tenfold more potent than was the cell-binding domain in inducing both cell types to invade SU-ECM basement membranes.

PHSRN was also found to be sufficient to stimulate keratinocyte and fibroblast chemotaxis in serum-free motility chamber assays. Cells were prebound to $2 \mu \mathrm{M}$ PHSRN and then placed in gelatin-coated motility chambers containing $2 \mu \mathrm{M}$ PHSRN in the top or in the bottom, and incubated for 12 hours. As shown in Figure 5, $2 \mu \mathrm{M}$ PHSRN peptide in the lower chambers stimulated the chemotactic migration of PHSRN-pretreated or BSA-pretreated keratinocytes and fibroblasts by 8to 30 -fold over $0.2 \%$ BSA treatment. Because two to more than ten standard deviations separated the means, the results are statistically significant to more than $95 \%$ confidence. Chemokinesis was negligible, as shown by the observation that the presence of $2 \mu \mathrm{M}$ PHSRN peptide in the upper chambers failed to stimulate migration to lower chambers by either of the cell types tested. Also, PHSRN pretreatment alone was not sufficient to stimulate the chemotactic migration observed, suggesting that continuous stimulation is required. PHSRN prebinding of the cells was shown to be unnecessary to observe chemotaxis toward PHSRN in the lower chambers, because very similar results demonstrating PHSRN-stimulated chemotaxis were obtained when cells were prebound with $0.2 \% \mathrm{BSA}$, also suggesting the need for continuous stimulation.

Effects of Ac-PHSRN-NH $2, A c-H S P N R-\mathrm{NH}_{2}$, and NS on reepithelialization in $d b / d b$ and $d b /+$ mice. Figure 6 summarizes the effects of a single topical application of AcPHSRN-NH $\mathrm{N}_{2}$ or Ac-HSPNR-NH $\mathrm{N}_{2}$ in NS compared to the effects of NS alone on the healing of identically sized dermal wounds of age-matched, healingimpaired, diabetic C57BL6/KsJ $d b / d b$ mice or of their age-matched, normally healing, nondiabetic $d b /+$ littermates. Neither blood nor lymph was evident in the dermal wounds of either $d b / d b$ or $d b /+$ mice for at least ten minutes after wounding, suggesting that the peptides applied were not significantly diluted by body fluids before being absorbed into each wound. Provisional matrix was not removed from the wounds during healing; however, at the time of complete reepithelialization of the treated wounds, the scabs were removed from representative untreated wounds to verify that their areas were not being significantly overestimated.

As shown in Figure 6a, one Ac-PHSRN-NH $\mathrm{H}_{2}$ treatment of $d b / d b$ wounds stimulated complete reepithelialization of all wounds in 8 days. This occurred whether the neighboring wound on each mouse was treated with AcPHSRN-NH ${ }_{2}$ or only with NS (untreated). Because all AcPHSRN-NH${ }_{2}$-treated $d b / d b$ wounds remained closed thereafter, healing appeared to be effective. Complete reepithelialization of all Ac-PHSRN-NH$H_{2}$-treated $d b /+$ nondiabetic wounds also occurred in 8 days. Complete reepithelialization of all untreated $d b /+$ wounds occurred

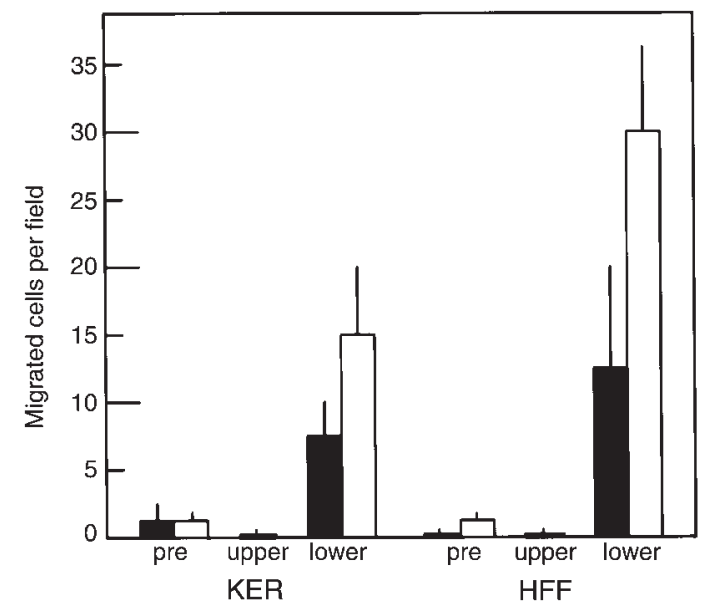

Figure 5

Effect of PHSRN on keratinocyte and fibroblast motility in Boyden chambers. X-axis shows cell types and chamber contents. KER, human keratinocytes. Exposure to PHSRN: pre, cells prebound with $1 \mu \mathrm{g} / \mathrm{mL}$ PHSRN (solid bars) or to $0.2 \%$ BSA (open bars); upper, cells exposed to $1 \mu \mathrm{g} / \mathrm{mL}$ PHSRN by both prebinding and by placement in upper chambers containing the same PHSRN concentration; lower, cells prebound to $1 \mu \mathrm{g} / \mathrm{mL}$ PHSRN (solid bars) or to $0.2 \%$ BSA (open bars) during prebinding, then placed in upper chambers containing $0.2 \%$ $\mathrm{BSA}$, and allowed to undergo chemotaxis in response to $1 \mu \mathrm{g} / \mathrm{mL}$ PHSRN in the lower chambers. Data presented as mean \pm SD. 


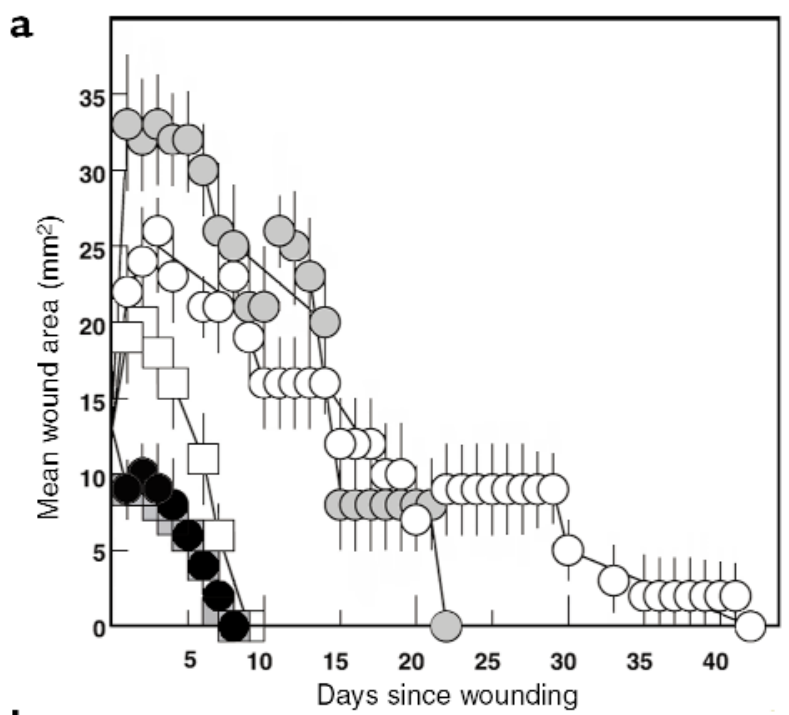

b

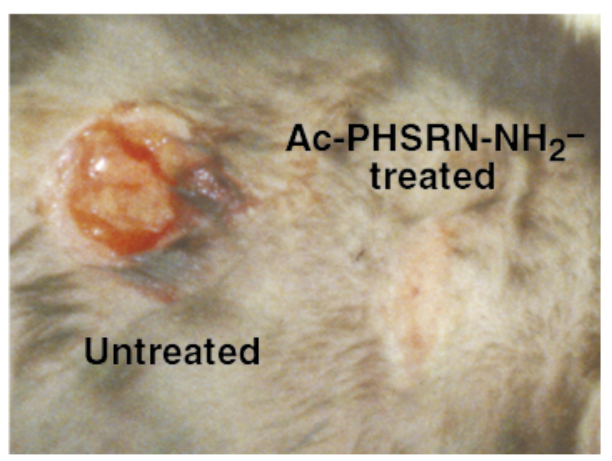

\section{Figure 6}

Functional activities of Ac-PHSRN-NH $H_{2}$ or Ac-HSPNR-NH $\mathrm{NH}_{2}$ in $d b / d b$ and in $d b /+$ dermal wounds. (a) Areas of dermal wounds treated with Ac-PHSRN-NH $\mathrm{H}_{2}, \mathrm{Ac}-\mathrm{HSPNR}-\mathrm{NH}_{2}$, or NS during the days required for wound closure. White circles, untreated $d b / d b$ wounds; black circles, Ac-PHSRN-NH ${ }_{2}$-treated $d b / d b$ wounds; gray circles, Ac-HSPNR$\mathrm{NH}_{2}$-treated $d b / d b$ wounds; white squares, untreated $d b /+$ wounds; gray squares, Ac-PHSRN-NH $\mathrm{N}_{2}$-treated $d b /+$ wounds. (b) Ac-PHSRN$\mathrm{NH}_{2}$-treated and untreated duplicate wounds in a $d b / d b$ mouse 7 days after wounding. Data presented as mean \pm SD.

slightly more slowly, but was complete in 9 days. Unlike Ac-PHSRN-NH $\mathrm{N}_{2}$-treated wounds, untreated wounds in $d b / d b$ mice took a long time to reepithelialize, and the time required for their closure varied widely. Wound closure time ranged from 20 days to 42 days, irrespective of whether the neighboring wound on each mouse was treated with NS or with Ac-PHSRN-NH ${ }_{2}$. A typical example comparing duplicate dermal wounds in a $d b / d b$ mouse treated with Ac-PHSRN-NH 2 or with NS alone is shown in Figure 6b. The scab was removed from the NStreated wound to demonstrate its lack of reepithelialization. The Ac-HSPNR-NH ${ }_{2}$-treated wounds of $d b / d b$ mice also required a very long time to complete reepithelialization (14-22 days), suggesting that the stimulation of $d b / d b$ wound healing by Ac-PHSRN-NH $\mathrm{N}_{2}$ was sequencespecific. Also, no Ac-HSPNR-NH ${ }_{2}$-treated or NS-treated $d b / d b$ wound completed reepithelialization before any AcPHSRN-NH ${ }_{2}$-treated wound. Thus, whereas Ac-PHSRN-
$\mathrm{NH}_{2}$ treatment significantly stimulated wound reepithelialization with respect to Ac-HSPNR-NH ${ }_{2}$ - or NS-treat$\mathrm{ed} \mathrm{db} / \mathrm{db}$ mice, there appeared to be no significant difference in the times required for wound closure among Ac-HSPNR-NH2-treated and untreated $d b / d b$ mice.

Interestingly, the areas of all Ac-PHSRN- $\mathrm{NH}_{2}$-treated wounds began to decrease immediately, suggesting that this peptide might attract cells or activate a pathway functioning in wound contraction. This was apparent even when the rates of closure of NS-treated $d b /+$ wounds were compared with those of Ac-PHSRN-NH${ }_{2}$-treated $d b / d b$ and Ac-PHSRN-NH ${ }_{2}$-treated $d b /+$ wounds.

The distance migrated by keratinocytes was measured microscopically in sectioned Ac-PHSRN- $\mathrm{NH}_{2}$-treated, Ac-HSPNR-N $\mathrm{H}_{2}$-treated, and NS-treated $d b / d b$ wounds during the 8 days after wounding. In this experiment, $24 \mathrm{db} / \mathrm{db}$ mice were wounded in duplicate and divided into three equally sized treatment groups. The wounds of one group of mice was treated with $2 \mu \mathrm{g}$ Ac-PHSRN$\mathrm{NH}_{2}$ in $5 \mu \mathrm{L}$ NS immediately after wounding. Another group was treated with the same dosage of Ac-HSPNR$\mathrm{NH}_{2}$. The wounds of a third (untreated) group received $5 \mu \mathrm{L}$ of NS only. On each of the 8 days after wounding, an arbitrarily chosen mouse from each group was sacrificed. The tissues bearing the wounds, dissected with surrounding skin intact, were subjected to histological analysis. Five sets of three serial sections, spanning a total of $1,325 \mu \mathrm{m}$ of wounded tissue, were cut from a central region of each wound and measured. For the 24 mice, Figure $7 \mathrm{a}$ shows the mean percentage reepithelialized when the 15 sections of each wound were measured for each of the 8 days after wounding. On days 7 and 8, the Ac-PHSRN-NH ${ }_{2}$-treated wounds were $100 \%$ reepithelialized upon gross examination and in all 15 sections measured, although the thickness of the new epidermis varied from multiple layers at the edges to about three layers in the centers of the wounds. In contrast, the migrating sheets of keratinocytes were much thinner and had only extended into $15-25 \%$ of the wound areas of the Ac-HSPNR- $\mathrm{NH}_{2}$-treated and the NS-treated $d b / d b$ wounds by this time. Because keratinocyte migration is not believed to depend on proliferation (44), the rapid reepithelialization of Ac-PHSRN$\mathrm{NH}_{2}$-treated wounds was probably due to the stimulation of migration. However, because AcPHSRN-NH $\mathrm{N}_{2}$-treated wounds appeared to decrease in size shortly after wounding, reepithelialization was also aided by the shorter distance required to be crossed. Figure $7 \mathrm{~b}$ shows a typical section of a hematoxylin and eosin-stained Ac-PHSRN-NH ${ }_{2}$-treated $d b / d b$ wound 8 days after wounding. A stratified layer of keratinocytes extends across the entire wound. Under the new epidermis is granulation tissue with numerous capillaries and many active fibroblasts, as judged by their extensive basophilic cytoplasm, euchromatic nuclei, and prominent nucleoli. Figure $7 \mathrm{c}$ shows a typical section of a stained Ac-HSPNR-NH $\mathrm{N}_{2}$-treated $d b / d b$ wound 8 days after wounding, with the ingrowing keratinocytes indicated. The provisional matrix has much more limited 

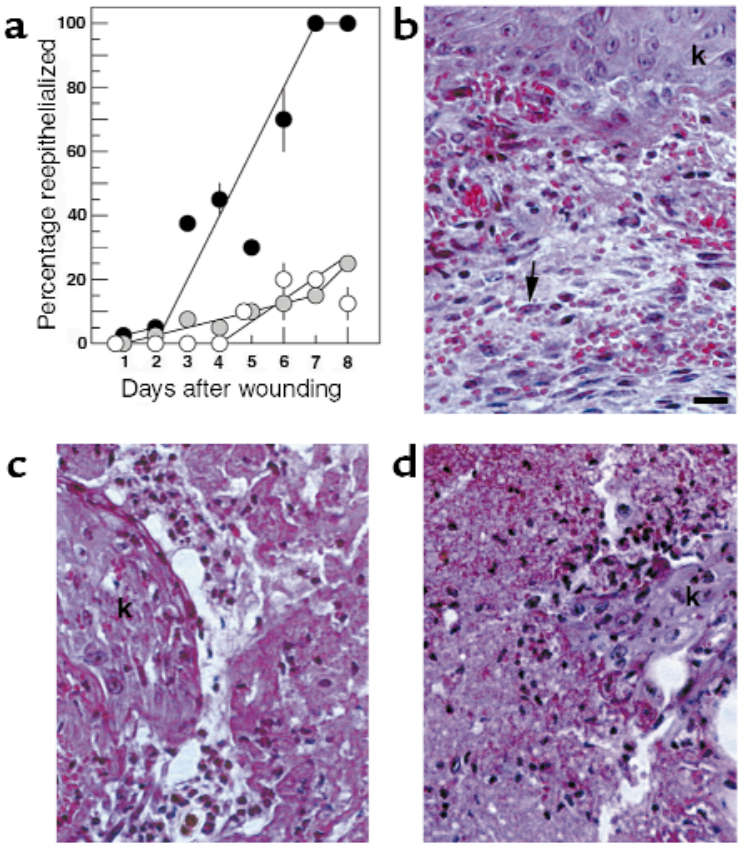

\section{Figure 7}

Effect of Ac-PHSRN-NH $\mathrm{H}_{2}$, Ac-HSPNR-NH $\mathrm{N}_{2}$, or NS alone on reepithelialization and fibroplasia in $d b / d b$ dermal wounds. (a) Effect of AcPHSRN-NH$H_{2}, A c-H S P N R-N_{2}$, or NS on reepithelialization. Y-axis shows mean percentage (with $\mathrm{SD}$ ) of wounds in section covered by keratinocytes. Black circles, mean percentage of each Ac-PHSRN$\mathrm{NH}_{2}$-treated wound reepithelialized; gray circles, mean percentage of each Ac-HSPNR- $\mathrm{NH}_{2}$-treated wound reepithelialized; white circles, mean percentage of each NS-treated wound reepithelialized. (b) A section of an Ac-PHSRN-NH $\mathrm{N}_{2}$-treated $d b / d b$ wound 8 days after wounding, photographed at $\times 400$ using phase-contrast optics. Arrow, active fibroblast; k, keratinocytes. Bar, $20 \mu \mathrm{m}$. (c) An Ac-HSPNR-NH ${ }_{2}$-treated $d b / d b$ wound 8 days after wounding shown at the same magnification as $\mathbf{b}$; $k$, keratinocytes. (d) A section of an untreated $d b / d b$ wound 8 days after wounding shown at the same magnification as $\mathbf{b}$. Identical areas of wound sections are shown in $\mathbf{b}, \mathbf{c}$, and $\mathbf{d}$.

vascularization and few active fibroblasts at this stage. Figure $7 \mathrm{~d}$ shows a section of an NS-treated $d b / d b$ wound 8 days after wounding, with the ingrowing keratinocytes indicated. The provisional matrix is not well vascularized, and few active fibroblasts are visible.

The provisional matrix of sectioned wounds in $d b / d b$ mice after wounding and treatment with Ac-PHSRN$\mathrm{NH}_{2}$, Ac-HSPNR-NH $\mathrm{H}_{2}$, or NS was also examined microscopically for the presence of fibroblasts. As shown in Figure 8a, by 3 days after wounding, the provisional matrix deep in Ac-PHSRN-NH${ }_{2}$-treated wounds contained numerous activated fibroblasts and prominent fibers, arranged in layers parallel to the wound surface and extending down to the base of the wound. The fibers and fibroblasts are shown at a higher magnification in Figure 8b. The provisional matrix of this AcPHSRN-NH${ }_{2}$-treated wound also appeared to contain macrophages, another very important cell in wound healing (23). The arrangement of fibroblasts and fibers observed has been interpreted as characteristic of a later stage in granulation tissue formation (reviewed in refs. 1 and 45), and was consistent with the contraction of Ac-PHSRN-NH $\mathrm{N}_{2}$-treated wounds occurring at this time, as shown in Figure 6a. In contrast to the AcPHSRN- $-\mathrm{NH}_{2}$-treated wounds, the provisional matrix of the NS-treated wounds 3 days after wounding exhibited more limited provisional matrix, fewer fibroblasts and fibers, and many more neutrophils, as shown in Figure 8c. Consistent with the sequence specificity of the effects of Ac-PHSRN-NH$H_{2}$ treatment, the fibroblasts and fibers were also much less conspicuous in the provisional matrix of the Ac-HSPNR- $\mathrm{NH}_{2}$-treated wounds, as shown in Figure 8d, whereas neutrophils were much more prevalent. These features have been viewed as characteristic of the earlier inflammatory stage of cutaneous wound repair (1).

\section{Discussion}

Nanomolar to micromolar concentrations of the PHSRN peptide induce keratinocytes and fibroblasts placed on the exterior surfaces of SU-ECM invasion substrates $(25,28)$ to migrate across the ectodermal extracellular matrix and the underlying lamina lucida of the basement membrane before migrating across its lamina densa (46) to the blastocelic cavity. Although it is not necessary for these cells to cross the basement membrane to enter the wound provisional matrix, enzymatic features of migration across the basement membrane in this direction may be similar to those involved
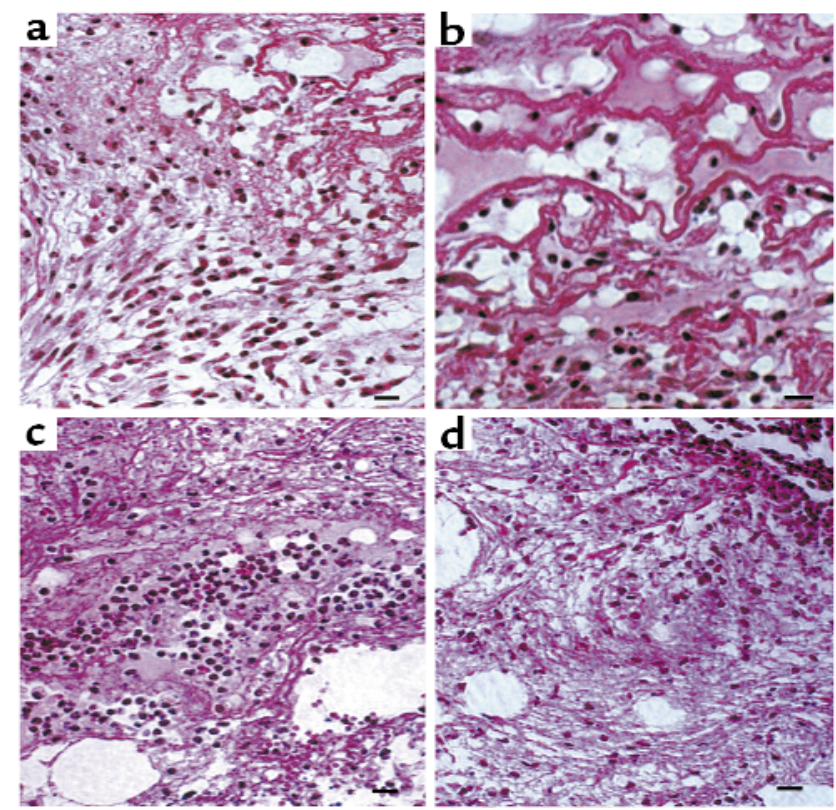

\section{Figure 8}

Effect of Ac-PHSRN-NH ${ }_{2}$, Ac-HSPNR-NH $\mathrm{N}_{2}$, or NS alone on the presence of fibroblasts in the provisional matrix of $d b / d b$ dermal wounds 3 days after wounding. (a) A section of an Ac-PHSRN-NH -treated wound in a $d b / d b$ mouse 3 days after wounding, Bar, $20 \mu \mathrm{m}$. (b) A section of an Ac-PHSRN-NH -treated wound in a $d b / d b$ mouse 3 days after wounding. Bar, $20 \mu \mathrm{m}$. (c) A section of an NS-treated wound in a $d b / d b$ mouse 3 days after wounding, Bar, $20 \mu \mathrm{m}$. (d) A section of an Ac-HSPNR-NH $\mathrm{H}_{2}$-treated wound in a $d b / d b$ mouse 3 days after wounding. Bar, $20 \mu \mathrm{m}$. a-d, $\times 400$. 
in invading the provisional matrix, in that both processes may involve the activation of interstitial collagenase (11, 14, and data not shown). The PHSRN sequence thus appears to induce invasion through interaction with the $\alpha 5 \beta 1$ integrin fibronectin receptor by a mechanism distinct from that of its previously defined function as a synergy sequence during $\alpha 5 \beta 1$ adhesion to the substrate RGD sequence $(40,41)$. This suggests that the mediation of adhesion and migration by the $\alpha 5 \beta 1$ receptor is a complex process. The invasion-inducing activity of PHSRN and of its more active derivative, AcPHSRN-NH ${ }_{2}$, have also been observed for other types of epithelial cells in vitro, including normal human prostate (28) and mammary epithelial cells (D. Livant et al., manuscript in preparation). The PHSRN peptide is sufficiently active that nearly all of the invasion-inducing activity of the cell-binding domain appears to be present in this sequence. Moreover, Ac-PHSRN-NH$H_{2}$ is 100 -fold more active than is PHSRN when tested in vitro, perhaps because the removal of terminal charges allows the peptide to assume a more active conformation, and increases its resistance to exoproteinases.

Diabetes is a chronic disorder of glucose homeostasis that affects $6 \%$ of the general population. Diabetics exhibit delayed wound healing (47), probably because of changes in vascular basement membranes and extracellular matrix that may delay the entry of cells into wounds (reviewed in ref. 48). Thus, topical agents with structures related to that of Ac-PHSRN- $\mathrm{NH}_{2}$ could be of significant therapeutic value. The genetically obese diabetic C57BL/KsJ $d b / d b$ mouse is a well-known and clinically relevant model of impaired wound healing (35-38). The defect in tissue repair has been related to a delay in cellular entry (37), making this an attractive model in which to test the effects of the Ac-PHSRN-NH $\mathrm{H}_{2}$ peptide.

Consistent with its invasion-inducing activity in vitro, a single dose of the Ac-PHSRN-NH 2 peptide stimulates keratinocyte and fibroblast migration into $d b / d b$ wounds, although its effect might have been minimized in our experiments because the histidine residue is replace by proline in mice (reviewed in ref. 49). Thus, Ac-PHSRN-NH$H_{2}$ treatment appears to enhance the migration-dependent processes of reepithelialization, fibroplasia, and neovascularization in the dermal wounds of healing-impaired $d b / d b$ mice. It also appears to enhance wound contraction, and to stimulate the layered arrangement of fibroblasts and fibers normally associated with it (reviewed in ref. 45). As a result of its stimulation, the time required for the closure of all Ac-PHSRN-NH${ }_{2}$-treated dermal wounds in $d b / d b$ mice is markedly decreased compared with Ac-HSPNR-NH $\mathrm{N}_{2}$-treated or NS-treated wounds. Because Ac-PHSRN-NH$H_{2}$-treated $d b / d b$ wounds reepithelialize slightly faster than do identical, untreated wounds in their normal $d b /+$ littermates, and as rapidly as Ac-PHSRN-NH wounds in $d b /+$ mice, topical treatment with AcPHSRN-NH$H_{2}$ may substantially reduce or eliminate the wound healing defect in $d b / d b$ mice.
On a molar basis, Ac-PHSRN-NH$H_{2}$ appears to be a more active inducer of invasion than the entire $\mathrm{pFn}$ cell-binding domain by at least tenfold. This suggests the interesting possibility that the production of active PHSRN sequence in wounds may be a proximal event for the stimulation of migration through a variety of extracellular matrices, and that active PHSRN sequence production may be driven by mechanisms that are defective in diabetic mice. There is precedence for peptides with significantly increased effects on adhesion, motility, and chemotaxis, which derive from extracellular matrix proteins such as type IV collagen (50) and laminin (51-53). There are also numerous examples of small active peptide hormones that are cleaved from large, inactive precursors (reviewed in ref. 54).

Thus, pFn may be a source of a locally acting peptide chemotactic factor, which functions to attract the cells that are crucial for wound healing by inducing chemotaxis through the stroma and directional migration on the provisional matrix. The stimulation of cell migration by diffusable cytokines, which form concentration gradients from their source tissues, is very important in wound healing and host defense. Many chemotactic cytokines function in inflammation to recruit monocytes, neutrophils, or fibroblasts to wounds (reviewed in ref. 55). Often, these molecules are generated by proteolytic cleavage from inactive, soluble precursors after wounding (56). Considering $\mathrm{pFn}$ as a source of a potent, locally active chemotactic factor suggests that the cleavage of other soluble extracellular matrix molecules may also generate cytokines regulating chemotaxis and invasion into wounds.

\section{Acknowledgments}

This work was supported in part by the National Institute of Diabetes and Digestive and Kidney Diseases of the National Institutes of Health (NIH; 5p60DK20572), a Basil O'Connor Award from the March of Dimes, an award from the entitled grant "SPORE in Prostate Cancer" (1P50CA69568), an award from the Office of the Vice President for Research at the University of Michigan (to D.L. Livant), and NIH grant HL53713 (to K. Kurachi). D.L. Allen is the recipient of a postdoctoral training fellowship from the University of Michigan Cardiovascular Research Center.

\footnotetext{
1. Clark, R.A.F. 1996. Wound repair: overview and general considerations. In The molecular and cellular biology of wound repair. 2 nd edition. R.F. Clark, editor. Plenum Press. New York, New York, USA. 3-50.

2. Martin, P. 1997. Wound healing: aiming for perfect skin regeneration. Science. 276:75-81.

3. Mosher, D.F. 1984. Physiology of fibronectin. Annu. Rev. Med. 35:561-575.

4. Clark, R.A.F., et al. 1982. Fibronectin and fibrin provide a provisional matrix for epidermal cell migration during wound reepithelialization. J. Invest. Dermatol. 70:264-269.

5. Clark, R.A.F., Quinn, J.H., Winn, H.J., and Colvin, R.B. 1983. Fibronectin beneath reepithelializing epidermis in vivo: sources and significance. $J$. Invest. Dermatol. 80(Suppl.):26S-30S.

6. Grinnell, F., Billingham, R.E., and Burgess, L. 1981. Distribution of fibronectin during wound healing in vivo. J. Invest. Dermatol. 76:181-189.

7. Clark, R.A.F., Wikner, N.E., Doherty, D.E., and Norris, D.A. 1988. Cryptic chemotactic activity of fibronectin for human monocytes resides in the 120-kDa fibroblastic cell-binding fragment. J. Biol. Chem. 263:12115-12123.
} 
8. Kim, L.T., et al. 1992. Integrin receptors and RGD sequence in human keratinocyte migration: unique anti-migratory function of the $\alpha 3 \beta 1$ epiligrin receptor. J. Invest. Dermatol. 98:764-770.

9. Oda, K., et al. 1992. Immunohistochemical study of transforming growth factor beta, fibronectin, and fibronectin receptor in invasive mammary carcinomas. Acta Pathol. Jpn. 42:645-650.

10. Roklin, O.W., and Cohen, M.B. 1995. Expression of cellular adhesion molecules on human prostate tumor cell lines. Prostate. 26:205-212.

11. Huhtala, P., et al. 1995. Cooperative signaling by $\alpha 5 \beta 1$ and $\alpha 4 \beta 1$ integrins regulates metalloproteinase gene expression in fibroblasts adhering to fibronectin. J. Cell Biol. 129:867-879.

12. Larjava, H., Salo, T., Haapasalmi, K., Kramer, R.H., and Heino, J. 1993 Expression of integrins and basement membrane components by wound keratinocytes. J. Clin. Invest. 92:1425-1435.

13. Cavani, A., et al. 1993. Distinctive integrin expression in the newly forming epidermis during wound healing in humans. J. Invest. Dermatol. 101:600-604.

14. Saarialho-Kere, U.K., et al. 1995. Interstitial collagenase is expressed by keratinocytes that are actively involved in reepithelialization in blistering skin disease. J. Invest. Dermatol. 104:982-988.

15. O'Keefe, E.J., Payne, R.E., Jr., Russell, N., and Woodley, D.T. 1985. Spreading and enhanced motility of human keratinocytes on fibronectin. J. Invest. Dermatol. 85:125-130.

16. Greiling, D., and Clark, R.A.F. 1997. Fibronectin provides a conduit for fibroblast transmigration from collagenous stroma into fibrin clot provisional matrix. J. Cell Sci. 110:861-870.

17. Grinnell, F., and Zhu, M. 1994. Identification of neutrophil elastase as the proteinase in burn wound fluid responsible for degradation of fibronectin. J. Invest. Dermatol. 103:155-161.

18. Grinnell, F., Ho, C.H., and Wysocki, A.J. 1992. Degradation of fibronectin and vitronectin in chronic wound fluid: analysis by cell blotting, immunoblotting, and cell adhesion assays. J. Invest. Dermatol. 98:410-416.

19. Postlethwaite, A.E., Keski-Oja, J., Balian, G., and Kang, A. 1981. Induction of fibroblast chemotaxis by fibronectin. Location of the chemotactic region to a 140,000 molecular weight nongelatin binding fragment. J. Exp. Med. 153:494-499.

20. Seppa, H.E., et al. 1981. The cell-binding fragment of fibronectin is chemotactic for fibroblasts. Cell Biol. Int. Rep. 5:13-19.

21. Adelmann-Grill, B.C., Wach, F., Behr, J., and Krieg, T. 1989. Involvement of protein kinase $\mathrm{C}$ in signal transduction during fibroblast chemotaxis to platelet-derived growth factor and a fragment of fibronectin. Eur. J. Cell Biol. 50:128-131.

22. Mignatti, P., Rifkin, D.B., Welgus, H.G., and Parks, W.C. 1996. Proteinases and tissue remodeling. In The molecular and cellular biology of wound repair. 2nd edition. R.F. Clark, editor. Plenum Press. New York, New York, USA. 427-474

23. Doherty, D.E., Henson, P.M., and Clark, R.A.F. 1990. Fibronectin fragments containing the RGDS cell-binding domain mediate monocyte migration into the rabbit lung. J. Clin. Invest. 86:1065-1075.

24. Grant, M.B., Caballero, S., Bush, D.M., and Spoerri, P.E. 1998 Fibronectin fragments modulate human retinal capillary cell proliferation and migration. Diabetes. 47:1335-1340.

25. Livant, D.L., Linn, S., Markwart, S., and Shuster, J. 1995. Invasion of selectively permeable sea urchin embryo basement membranes by metastatic tumor cells, but not by their normal counterparts. Cancer Res. 55:5085-5093.

26. Noel, J.C., et al. 1991. Invasion of reconstituted basement membrane matrix is not correlated to the malignant metastatic cell phenotype. Can cer Res. 51:405-414.

27. Simon, N., Noel, A., and Foidart, J.M. 1992. Evaluation of in vitro reconstituted basement membrane assay to assess the invasiveness of tumor cells. Invasion Metastasis. 12:156-167.

28. Livant, D.L., et al. 2000. Anti-invasive, anti-tumorigenic, and antimetastatic activities of the PHSCN sequence in prostate carcinoma. Can cer Res. 60:309-320.

29. Litvinovich, S.V., and Ingham, K.C. 1995. Interactions between type III domains in the $110 \mathrm{kDa}$ cell-binding fragment of fibronectin. J. Mol. Biol. 248:611-626

30. Wayner, E.A., Carter, W.G., Piotrowicz, R.S., and Kunicki, T.J. 1988. The function of multiple extracellular matrix receptors in mediating cell adhesion to extracellular matrix: preparation of monoclonal antibodies to the fibronectin receptor that specifically inhibit cell adhesion to fibronectin and react with platelet glycoproteins Ic-IIa. J. Cell Biol. 107:1881-1891

31. Carter, W.E., Wayner, E.A., Bouchard, T.S., and Kaur, P. 1990. The role of integrins $\alpha 2 \beta 1$ and $\alpha 3 \beta 1$ in cell-cell and cell-substrate adhesion of human epidermal cells. J. Cell Biol. 110:1387-1404.

32. Atherton, E., and Sheppard, R.C. 1989. Solid phase peptide synthesis: a prac- tical approach. IRL Press. Eynsham, Oxford, United Kingdom. 25-37

33. Mant, C.T., Kondejewski, L.H., Cachia, P.J., Monera, O.D., and Hodges, R.S. 1997. Analysis of synthetic peptides by high-performance liquid chromatography. Methods Enzymol. 289:426-469.

34. Postlethwaite, A.E., Snyderman, R., and Kang, A.H. 1976. The chemotactic attraction of human fibroblasts to a lymphocyte-derived factor. J. Exp. Med. 144:1188-1203.

35. Tsuboi, R., and Rifkin, D.B. 1990. Recombinant basic fibroblast growth factor stimulates wound healing in healing-impaired $d b / d b$ mice. J. Exp. Med. 172:245-251.

36. Greenhalgh, D.G., Sprugel, K.H., Murray, M.J., and Ross, R. 1990. PDGF and FGF stimulate wound healing in the genetically diabetic mouse. Am. J. Pathol. 136:1235-1246.

37. Greenhalgh, D.G., Hummel, R.P., III, and Albertson, S. 1993. Synergistic actions of platelet-derived growth factor and the insulin-like growth factors in vivo: enhancement of tissue repair in genetically diabetic mice. Wound Repair Regen. 1:69-82.

38. Brown, R.L., Breeden, M.P., and Greenhalgh, M.D. 1994. PDGF and TGF- $\alpha$ act synergistically to improve wound healing in the genetically diabetic mouse. J. Surg. Res. 56:562-570.

39. Humason, G.L. 1972. Animal tissue techniques. W, H. Freeman and Co. SanFrancisco, California, USA. 34-169.

40. Aota, S., Nomizu, M., and Yamada, K.M. 1994. The short amino acid sequence Pro-His-Ser-Arg-Asn in human fibronectin enhances cell-adhesive function. J. Biol. Chem. 269:24756-24761.

41. Aota, S., Nagai, T., and Yamada, K.M. 1991. Characterization of regions of fibronectin besides the arginine-glycine-aspartic acid sequence required for adhesive function of the cell-binding domain using sitedirected mutagenesis. J. Biol. Chem. 266:15938-15943.

42. Mould, A.P., et al. 1997. Defining the topology of integrin alpha5beta1fibronectin interactions using inhibitory anti-alpha5 and anti-beta1 monoclonal antibodies. Evidence that the synergy sequence of fibronectin is recognized by the amino-terminal repeats of the alpha 5 subunit. J. Biol. Chem. 272:17283-17292.

43. Gailit, J., and Clark, R.A. 1996. Studies in vitro on the role of alpha $v$ and beta 1 integrins in the adhesion of human dermal fibroblasts to provisional matrix proteins fibronectin, vitronectin, and fibrinogen. J. Invest. Dermatol. 106:102-108.

44. Sarret, Y., Woodley, D.T., Grigsby, K., Wynn, K.C., and O'Keefe, E.J. 1992. Human keratinocyte locomotion: the effect of selected cytokines. $J$. Invest. Dermatol. 98:12-16.

45. MacSween, R.N.M., and Whaley, K. 1992. Inflammation, healing and repair. In Muir'stextbook of pathology. 13th edition. R.N.M. Mac Sween and K. Whaley, editors. Edward Arnold. London, United Kingdom. 112-165.

46. Amemiya, S. 1989. Development of the basal lamina and its role in migration and pattern formation of primary mesenchyme cells in sea urchin embryos. Dev. Growth Differ. 31:131-145.

47. Harris, M., Hadden, W.C., Knowles, W.C., and Bennett, P.H. 1987. Prevalence of diabetes and impaired glucose tolerance and glucose levels in the US population ages 20-40 years. Diabetes. 36:523-534.

48. Williamson, J.R., Tilton, R.G., Chang, K., and Kilo, C. 1988. Basement membrane abnormalities in diabetes mellitus: relationship to clinical microangiopathy. Diab. Metabol. Rev. 4:339-370.

49. Akiyama, S.K., Older, K., and Yamada, K.M. 1995. Fibronectin and integrins in invasion and metastasis. Cancer Metastasis Rev. 14:173-189.

50. Chelberg, M.K., McCarthy, J.B., Skubitz, A.P., Furcht, L.T., and Tsilibary, E.C. 1990. Characterization of a synthetic peptide from type IV collagen that promotes melanoma cell adhesion, spreading, and motility. J. Cell Biol. 111:262-270

51. Graf, J., et al. 1987. Identification of an amino acid sequence in laminin mediating cell attachment, chemotaxis, and receptor binding. Cell. 48:989-996.

52. Graf, J., et al. 1987. A pentapeptide from the laminin B1 chain mediates cell adhesion and binds the 67,000 laminin receptor. Biochemistry. 26:6896-6900.

53. Richard, B.L., Nomizu, M., Yamada, Y., and Kleinman, H.K. 1996. Identification of synthetic peptides derived from laminin $\alpha 1$ and $\alpha 2$ chains with cell type specificity for neurite outgrowth. Exp. Cell Res. 228:98-105.

54. König, W. 1993. Corticoliberin-proopiomelanocortin cascade. In Peptide and protein hormones: structure, regulation, activity: a reference manual. VCH Verlagsgesellschaft, Weinheim, Germany, and VCH publishers, New York, New York, USA. 51-88

55. Miller, M.D., and Krangel, M.S. 1992. Biology and biochemistry of the chemokines: a family of chemotactic and inflammatory cytokines. Crit. Rev. Immunol. 12:17-46.

56. Walz, A., Dewald, B., von Tscharner, V., and Baggiolini, M. 1989. Effects of the neutrophil-activating peptide NAP-2, platelet basic protein, connective tissue-activating peptide III, and platelet factor 4 on human neutrophils. J. Exp. Med. 170:1745-1750. 